\title{
Definition of Derivative Function: Logical Error in Mathematics
}

\author{
Temur Z. Kalanov \\ Home of Physical Problems, Yozuvchilar (Pisatelskaya) 6a, 100128 Tashkent, Uzbekistan
}

\begin{abstract}
The critical analysis of the foundations of the differential calculus is proposed. Methodological basis of the analysis is the unity of formal logic and of rational dialectics. It is shown that differential calculus is fictitious mathematical theory because the concept of the limiting process is the starting point for definition of the derivative function. The passage to the limit "zero" in the definition of the derivative function signifies that the variable quantity takes the only essential value "zero". This fact leads to the following errors. (1) The definition of the derivative function is based on the violation of the necessary and sufficient condition for the validity of the relationship between the increment of the function argument and the increment of the function because the increment of the function is divided by the zero increment of the argument in the case of the limiting process. (2) The definition of the derivative function is based on the contradiction which is that the increment of the argument is both zero and not zero in the same relationship. This contradiction represents a violation of the formal-logical law of identity and of the formallogical law of the lack of contradiction. (3) The definition of the differential of function is based on two contradictory (mutually exclusive) features: the differential of the argument is not zero while the increment of the argument in the definition of the derivative function is zero.
\end{abstract}

Keywords: General mathematics; Foundations of mathematics; Differential calculus; Integral calculus; Methodology of mathematics; Philosophy of mathematics; Philosophy and mathematics; Mathematics education; Logic; Physics.

MSC: 00A30; 00A35; 03A05; 00A05; 00A69; 00A79; 97140, 97150; 97E20; 97E30; 97A99.

\section{(ㄷ) (1) CC BY: Creative Commons Attribution License 4.0}

\section{Introduction}

As is known, the formalism of differential and integral calculus is widely and successfully used in natural sciences. However, this does not mean that the problem of substantiation of differential and integral calculus is completely solved in 20-21 centuries, and the foundations of differential and integral calculus are not in need of formal-logical analysis now. Recently, necessity of the critical analysis of the foundations of differential and integral calculus within the framework of the correct methodological basis - unity of formal logic and of rational dialectics has arisen [1-14]. It is shown in Kalanov [1], Kalanov [2], Kalanov [3], Kalanov [4], Kalanov [5], Kalanov [6], Kalanov [7], Kalanov [8], Kalanov [9], Kalanov [10], Kalanov [11], Kalanov [12] ,Kalanov [13] ,Kalanov [14], that errors in science (for example, in physics) often arise because of the existence of methodological errors in mathematics and because of the "thoughtless application of mathematics" (L. Boltzmann).

The purpose of this work is to propose the critical analysis of the foundations of differential calculus within the framework of methodological basis - the unity of formal logic and of rational dialectics. The critical analysis is based on the dialectical principle of functional connection and of movement.

\section{The Principle of Functional Connection and of Movement}

Movement is Change in General In other words, movement is a change in state.

The principle of movement (change) is a theoretical generalization of practice and represents a concretization of the laws of dialectics and formal logic. The principle of functional connection and of movement in mathematics is formulated as follows.

a) If the continuous function $y$ of one argument $x$ is given, then the function

$$
y=f(x)
$$

is a mathematical (quantitative) representation of the dialectical principle of functional connection. The principle of quantitative change in the functional connection reads as follows: a change in the values of the argument $x$ leads to a change in the values of the function ${ }^{y}$; a change in the values of the function ${ }^{y}$ characterizes a change in the values of the argument $x$. (In other words, a change in the argument determines a change in the function; a change in the function characterizes a change in the argument).

b) The change in the values of the argument $x$ is characterized by the increment $\Delta x$ of the argument. The quantity $\Delta x$ takes certain numerical values. The definition of the argument increment is the following: the increment of the argument is the difference of the two numerical values of the argument. Therefore, the dimension of the quantity $\Delta x$ is identical to the dimension of the quantity $x$. 
c) The change in the numerical values of the function $y_{\text {is characterized by the increment }} \Delta y$ of the function. The quantity $\Delta y$ takes certain numerical values. The definition of the function increment is the following: the function increment is the difference of two numerical values of the function. Therefore, the dimension of the quantity $\Delta y$ is identical to the dimension of the quantity $y$.

d) The relationships $\Delta x \neq 0$ and $\Delta y \neq 0$ represent a necessary and sufficient condition for movement (change). The relationships $\Delta x=0$ and $\Delta y=0$ represent a necessary and sufficient condition for the lack of movement (i.e., the condition for the lack of change).

e) The coefficient $k$ of the relative increment (i.e., the ratio of the quantity of the increment of the function to the quantity of the increment of the argument) is defined by the following relationship: $k \equiv \Delta y / \Delta x$ where the permitted values of the increments are in the regions $\Delta x \neq 0$ and $\Delta y \neq 0$. If the movement (change) is lack (that is, if $\Delta x=0$ and $\Delta y=0$ ), then the coefficient of relative increment loses its meaning: $k \equiv \Delta y / \Delta x=0 / 0$. In other words, the values $\Delta x=0$ and $\Delta y=0$ are inadmissible values in the calculation of $k \equiv \Delta y / \Delta x$.

f) The expression $\lim _{\Delta x \rightarrow q} \Delta x$ (where $q \neq 0$ is a given number in the region of existence of $\Delta x$ ) signifies that the values $\Delta x \neq q$ are nonessential values, and the value $\Delta x=q$ is essential one. Therefore, the values $\Delta x \neq q$ do not appear (do not show itself) in the expression (symbol) $\lim _{\Delta x \rightarrow q} \Delta x$. The value $\Delta x=q$ expresses the true meaning of the expression (symbol) $\lim _{\Delta x \rightarrow q} \Delta x$.

g) The expression $\lim _{\Delta x \rightarrow q} \Delta y$ (where $q \neq 0$ is a given number in the region of existence of $\Delta x$ ) signifies that the values $\Delta x \neq q$ are nonessential values, and the value $\Delta x=q$ is essential one. Therefore, the values $\Delta x \neq q$ do not appear (do not show itself) in the expression (symbol) $\lim _{\Delta x \rightarrow q} \Delta y$. The value $\lim _{\Delta x \rightarrow q} \Delta y=p$ (where $p$ is a number in the region of existence of $\Delta y$ ) expresses the true meaning of the expression (symbol) $\lim _{\Delta x \rightarrow q} \Delta y$

h) The true meaning of the expression (symbol) $\lim _{\Delta x \rightarrow q} \frac{\Delta y}{\Delta x}$ is the following:

$$
\lim _{\Delta x \rightarrow q} \frac{\Delta y}{\Delta x} \equiv \frac{\lim _{\Delta x \rightarrow q} \Delta y}{\lim _{\Delta x \rightarrow q} \Delta x}=\frac{p}{q}
$$

i) In the point of view of formal logic, the right and left sides of the mathematical relationship for $\Delta y / \Delta x$ must satisfy the formal-logical law of identity:

$$
\begin{aligned}
\left(\frac{(\text { concept of } \Delta y)}{(\text { concept of } \Delta x)}\right) & =\left(\frac{(\text { concept of } \Delta y)}{(\text { concept of } \Delta x)}\right) \\
\left(\frac{(\text { definition of concept of } \Delta y)}{(\text { definition of concept of } \Delta x)}\right) & =\left(\frac{\left(\text { definition of concept of } \lim _{\Delta x \rightarrow q} \Delta y\right)}{\left(\text { definition of concept of } \lim _{\Delta x \rightarrow q} \Delta x\right)}\right)
\end{aligned}
$$

j) In order to clarify the meaning of the above quantities and designations, one must concretize (specify) the quantities and designations. The quantities $x$ and $y$ take numerical values as a result of measurements (observations). The result of measurements (observations) of the quantity $x$ represents the following values: $x_{n}$, $n=0,1,2, \ldots$. These values correspond to the following result of measurements (observations) of quantity $y$ : $y_{n}, \quad n=0,1,2, \ldots$. In this case, the increments are designated as follows: $x_{n+1}-x_{n} \equiv \Delta x_{n+1, n}$, $y_{n+1}-y_{n} \equiv \Delta y_{n+1, n}$. Increments $\Delta x_{n+1, n}$ and $\Delta y_{n+1, n}$ represent the results of mathematical 
operations. In other words, increments $\Delta x_{n+1, n}$ and $\Delta y_{n+1, n}$ are numbers. If $x_{n+1}-x_{n}=h, h \neq 0$, then

$$
\begin{aligned}
& x_{n}=n h \\
& k_{n+1, n}=\frac{\Delta y_{n+1, n}}{\Delta x_{n+1, n}}=\frac{\Delta y_{n+1, n}}{h} \\
& \Delta y_{n+1, n}=k_{n+1, n} h, y_{n+1}=y_{n}+k_{n+1, n} h .
\end{aligned}
$$

These algebraic relationships express arithmetic relationships between numbers.

k) Proportion is the only correct relationship between changes in the values of the argument and of the function:

$$
\begin{aligned}
& \left(\frac{y(x)-y_{1}\left(x_{1}\right)}{y_{1}\left(x_{1}\right)}\right)=\left(\frac{x-x_{1}}{x_{1}}\right), \text { under } x-x_{1} \neq 0 \text {, } \\
& y(x)-y_{1}\left(x_{1}\right)=\frac{y_{1}\left(x_{1}\right)}{x_{1}}\left(x-x_{1}\right) \\
& y(x)=\frac{y_{1}\left(x_{1}\right)}{x_{1}} x \\
& \left(\frac{y(x+\Delta x)-y(x)}{y(x)}\right)=\left(\frac{x+\Delta x-x}{x}\right) \\
& y(x+\Delta x)-y(x)=\frac{y(x)}{x} \Delta x \\
& y(x+0)-y(x)=\frac{y(x)}{x} 0, \quad 0=\frac{y(x)}{x} 0,0=0 \text { under } \Delta x=0 \\
& \frac{y(x+\Delta x)-y(x)}{\Delta x}=\frac{y(x)}{x}, \text { under } \Delta x \neq 0, \\
& y(x+\Delta x)=\frac{y(x)}{x}(x+\Delta x) \\
& y(x)=\frac{y(x)}{x} x, y(x)=y(x) \text { under } \Delta x=0 \text {. }
\end{aligned}
$$

\section{Definition of the Derivative Function}

As is known [15, 16], if $y=f(x)$ represents the continuous function $y$ of one argument $x$, then the derivative function is defined as follows:

$$
\begin{aligned}
& y^{\prime}(x)=f^{\prime}(x) ; \\
& f^{\prime}(x) \equiv f^{\prime}(x ; \Delta x=0) \equiv \lim _{\Delta x \rightarrow 0} \frac{\Delta y}{\Delta x} \equiv \lim _{q \rightarrow 0}\left[\frac{\lim _{\Delta x \rightarrow q \neq 0} \Delta y}{\lim _{\Delta x \rightarrow q \neq 0} \Delta x}\right]
\end{aligned}
$$

where

$$
y^{\prime}(x) \neq \frac{\lim _{\Delta x \rightarrow 0} \Delta y}{\lim _{\Delta x \rightarrow 0} \Delta x}=\frac{0}{0} \quad \frac{\Delta y}{\Delta x}=\frac{f(x+\Delta x)-f(x)}{\Delta x}
$$

$\Delta x$ and $\Delta y=f(x+\Delta x)-f(x)$ are the increments of the argument and of the function, respectively.

As is known $[15,16]$, 


$$
\frac{d y}{d x}=f^{\prime}(x), d y=f^{\prime}(x) d x, d y=d f(x)
$$

where $d x=\Delta x \neq 0$ and $d y=\Delta y \neq 0$ are the differentials of the argument and of the function, respectively. The differential $d y$ is a function of two variable quantities $x$ and $d x$ which are independent of each other.

The essence of the concept of the derivative function becomes the apparent

(obvious, evident, certain) fact in the following example.

\section{Example}

If $y=x^{2}$, then

$$
\begin{gathered}
y^{\prime}=\lim _{\Delta x \rightarrow 0} \frac{(x+\Delta x)^{2}-x^{2}}{\Delta x}=\lim _{\Delta x \rightarrow 0} \frac{2 x \Delta x+(\Delta x)^{2}}{\Delta x}= \\
=\lim _{\Delta x \rightarrow 0}(2 x+\Delta x)=2 x+0=2 x,
\end{gathered}
$$

where the symbol $\lim _{\Delta x \rightarrow 0} \Delta x$ signifies that the quantity $\Delta x$ takes the only essential value $\Delta x=0$.

The result $y^{\prime}=2 x$ is not free of the following objections.

(a) If one divides both sides of the relationship

$$
\Delta y=(x+\Delta \mathrm{x})^{2}-x^{2}
$$

by $\Delta x \neq 0$, then one obtains the following equality:

$$
\frac{\Delta y}{\Delta x}=\frac{2 x \Delta x+(\Delta x)^{2}}{\Delta x}=2 x+\Delta x, \quad \Delta x \neq 0 .
$$

The condition $\Delta x \neq 0$ represents the necessary and sufficient condition of validity of this equality. In other words, the left and right sides of true equality must satisfy the condition $\Delta x \neq 0$. Therefore, the equality is not valid if $\Delta x=0$.

In this point of view, the relationship

$$
\lim _{\Delta x \rightarrow 0} \frac{\Delta y}{\Delta x}=\lim _{\Delta x \rightarrow 0} \frac{2 x \Delta x+(\Delta x)^{2}}{\Delta x}=\lim _{\Delta x \rightarrow 0}(2 x+\Delta x)=2 x
$$

contradicts to the condition $\Delta x \neq 0$. Consequently, the result $y^{\prime}=2 x$ is erroneous.

(b) The result $y^{\prime}=2 x$ is a consequence of the contradiction which is that $\Delta x \neq 0$ and $\Delta x=0$ in the same relationship.

Thus, the above example discovers (ascertains, reveals, detects) a formal-logical error in differential calculus.

\section{Logical Errors in the Definition of the Derivative Function}

To understand the essence (nature) of the error in the definition of the derivative function, one must know how the computer performs the calculations. As is known, a programmer and a computer perform the concretization of mathematical (quantitative) relationships expressed in terms of letters and symbols of operations. The computer cannot perform, for example, the operation of addition $x+\Delta x$ if the programmer does not set (specify) numerical values to the quantities $x$ and $\Delta x$. If the programmer sets (gives, specifies) numerical values to the quantities $x$ and $\Delta x$, then the computer can calculate the result of the mathematical operation. The computer does not distinguish between the quantities $\frac{\Delta y}{\Delta x}$ and $\lim _{\Delta x \rightarrow q} \frac{\Delta y}{\Delta x}$ because the computer operates only with 
numerical values that the programmer sets (gives, specifies) to mathematical quantities. The symbol $\begin{array}{ll}\lim \frac{\Delta y}{\Delta x \rightarrow q} & \frac{\Delta x}{\Delta x}\end{array}$ signifies that the quantity $\Delta x$ takes the only essential value $\Delta x=q$. Therefore, the computer divides the number $\Delta y \neq 0$ by the number $\Delta x \neq 0$ and gives the result in the form of the numerical fraction $\frac{p}{q}$. If $\Delta x=q=0$, then the computer gives the information that the specified division operation is the inadmissible operation $\frac{0}{0}$. Therefore, the condition $\Delta x \neq 0$ represents the necessary and sufficient condition of validity of the quantity $\frac{\Delta y}{\Delta x}$.

But in order to analyze and to understand why this condition is not satisfied in the definition of the derivative function, one must know the formal logic. The formal-logical analysis is not accessible to a computer because a computer cannot operate with concepts. Formal logic (as the science of the laws of correct thinking) operates with concepts and is accessible only to man.

The formal-logical errors in the definition of the derivative function are as follows.

1) In accordance with the law of identity, the object $\Delta x$ of thought must be

identical with itself in the process of reasoning: $\Delta x \equiv \Delta x$. But the definition of the derivative function $f^{\prime}(x)$ contains the contradiction which is that $\Delta x \neq 0$ and $\Delta x=0$ in the same relationship. This is a violation of the law of identity and the law of lack of contradiction.

2) In accordance with the law of lack of contradiction, it is not permitted that the same object of thought contains two contradictory features at the same time, in the same sense or in the same relation. But $d y=f^{\prime}(x) d x$ contains two contradictory features: $d x \neq 0$ and $\Delta x=0$ (in the definition of $f^{\prime}(x)$ ). The feature included in the content of the concept $d x \neq 0$ negates the feature included in the concept $\Delta x=0$ (in the definition of $f^{\prime}(x)$ ). One concept excludes another concept. But both features cannot belong to the same relationship. Therefore, one of two contradictory (inconsistent) features (or both) is a lie. Just because the feature $\Delta x=0$ is a lie.

Thus, differential calculus is a false theory because it contains formal-logical errors.

\section{Discussion}

1. The idea of mechanical movement played a "disgusting joke" with Isaac Newton. Newton entered (introduced) the concept of movement (change) into the mathematical expression of the function $y=f(x)$ by means of the increment $\Delta x$ of the argument $x$. He obtained the movement (change) $\Delta y$ of the function $y$. The "disgusting joke" is that Newton canceled (deleted) the change in the argument (i.e., he putted $\Delta x=0$ ), but, contrary to logic, he didn't canceled (he didn't deleted) the change in the function (i.e., he didn't put $\Delta y=0$ ). Newton was unable to detect the logical error because he could not understand the essence of the limiting process. (The essence of the limiting process $\lim _{\Delta x \rightarrow 0} \frac{\Delta y}{\Delta x}$ is that the quantity $\Delta x$ takes the only essential value ${ }^{0}$ ). As a result, Newton obtained $\Delta y \neq 0, f^{\prime}(x) \neq 0$ under $\Delta x=0$. This signifies that movement (or the cause of movement) does not exist (i.e., $\Delta x=0$ ), but such a feature (property) of movement as movement speed (i.e., derivative) exists. This is physical absurdity. Newton probably did not understand that the properties (speed, acceleration) of motion do not exist if motion does not exist. Thus, the absurdity in the form of differential and integral calculus entered in mathematics.

The absurdity took an elegant form (shape) thanks to the canon of differential calculus which was created by logician G. Leibniz. (For the first time, Leibniz's canon was published in the journal Acta Eruditorum, Leipzig, 1684). But Leibniz could not find, understand, and detect Newton's logical errors.

2. Today, mathematicians and physicists all over the world use differential and integral calculus. They believe in the correctness, firmness, and inviolability of this theory. Therefore, scientists do not work for mastery of the correct methodological basis of science: the unity of formal logic and of rational dialectics. The unity of formal logic and of rational dialectics is also a criterion of truth. But errors in science (for example, physics) 
often arise because of the existence of methodological errors in mathematics and the "mindless, thoughtless application of mathematics" (L. Boltzmann).

Is there "problem of existence of science for science" today? As the history of science shows, scientists are in no hurry to cast doubts on old theories within the framework of the correct criterion of truth because they are afraid to loss prestige and well-being.

\section{Conclusion}

Thus, the critical analysis of the foundations of differential calculus, carried out

within the framework of the correct methodological basis, leads to the following statements:

1) If the continuous function of one argument is given, then this function is a mathematical (quantitative) representation of the dialectical principle of the functional connection. The dialectical principle of the quantitative change in the functional connection is that a change (increment) in the argument leads to a change (increment) in the function.

2) The necessary and sufficient condition for the validity of the relation between the increment of the function argument and the increment of the function is that the increment of the argument must be non-zero in all cases. But this condition is violated in determination of the derivative function: in the case of the passage to limit "zero", the increment of the function is divided by the zero increment of the argument.

3) The definition of the derivative function contains the contradiction which is that the increment of the argument is both zero and non-zero in the same relationship. This contradiction represents a violation of the formal-logical law of identity and the formal-logical law of the lack of contradiction.

4) In accordance with the formally-logical law of the lack of contradiction, one and the same object of thought should not contain two contradictory features at the same time, in the same sense or in the same relation. But the definition of the differential of function contains two contradictory (mutually exclusive) features that cannot belong to the same relationship: the differential of the argument is not zero, but the increment of the argument is zero in the definition of the derivative function.

Thus, differential calculus is a fallacious mathematical theory because it contains formal-logical errors.

\section{References}

[1] Kalanov, T. Z., 2011. Critical analysis of the foundations of differential and integral calculus. MCMS, Ada Lovelace Publications, pp. 34-40.

[2] Kalanov, T. Z., 2011. "Logical analysis of the foundations of differential and integral calculus." Indian Journal of Science and Technology, vol. 4, pp.1789-1789

[3] Kalanov, T. Z., 2011. "Logical analysis of the foundations of differential and integral calculus." Bulletin of Pure and Applied Sciences, vol. 30, pp. 327-334.

[4] Kalanov, T. Z., 2012. "Critical analysis of the foundations of differential and integral calculus." International Journal of Science and Technology, vol. 1, pp. 80-84.

[5] Kalanov, T. Z., 2012. "On rationalization of the foundations of differential calculus." Bulletin of Pure and Applied Sciences (Math and Stat), vol. 31, pp. 1-7.

[6] Kalanov, T. Z., 2013. "Critical analysis of the mathematical formalism of theoretical physics. I. Foundations of differential and integral calculus." Bulletin of the Amer. Phys. Soc., vol. 58, pp. 1-4

[7] Kalanov, T. Z., 2015. "On the formal-logical analysis of the foundations of mathematics applied to problems in physics." Aryabhatta Journal of Mathematics and Informatics, vol. 7, pp. 1-2.

[8] Kalanov, T. Z., 2016. "On the formal-logical analysis of the foundations of mathematics applied to problems in physics." Bulletin of the Amer. Phys. Soc., vol. 5, pp. 25-30.

[9] Kalanov, T. Z., 2016. "Critical analysis of the foundations of pure mathematics." Mathematics and Statistics (CRESCO), vol. 2, pp. 2-14. Available: http://crescopublications.org

[10] Kalanov, T. Z., 2016. "Critical analysis of the foundations of pure mathematics." International Journal for Research in Mathematics and Mathematical Sciences, vol. 2, pp. 15-33.

[11] Kalanov, T. Z., 2016. "Critical analysis of the foundations of pure mathematics." Aryabhatta Journal of Mathematics and Informatics, vol. 8, pp. 1-14.

[12] Kalanov, T. Z., 2016. "Critical analysis of the foundations of pure mathematics." Philosophy of Mathematics Education Journal, vol. 2, pp. 19-36

[13] Kalanov, T. Z., 2017. "On the formal-logical analysis of the foundations of mathematics applied to problems in physics." Asian Journal of Fuzzy and Applied Mathematics, vol. 5, pp. 48-49.

[14] Kalanov, T. Z., 2017. The critical analysis of the foundations of mathematics. Mathematics: The art of scientific delusion. LAP LAMBERT Academic Publishing.

[15] Luzin, N. N., 1952. Differential calculus. Moscow.

[16] Smirnov, V. I., 1974. Course of higher mathematics. Moscow. 